\title{
B-type natriuretic peptides and mortality after stroke
}

\author{
A systematic review and meta-analysis
}

Teresa García-Berrocoso

Dolors Giralt

Alejandro Bustamante, MD

Thorleif Etgen, MD

Jesper K. Jensen, MD

Jagdish C. Sharma, FRCP

Kensaku Shibazaki, MD

Ayhan Saritas, MD

Xingyong Chen

William N. Whiteley, MRCP

Joan Montaner, PhD

Correspondence to

Dr. Montaner:

31862jmv@comb.es

Editorial, page 1970

Supplemental data at www.neurology.org

\section{ABSTRACT}

Objective: To measure the association of B-type natriuretic peptide (BNP) and N-terminal fragment of BNP (NT-proBNP) with all-cause mortality after stroke, and to evaluate the additional predictive value of BNP/NT-proBNP over clinical information.

Methods: Suitable studies for meta-analysis were found by searching MEDLINE and EMBASE databases until October 26, 2012. Weighted mean differences measured effect size; metaregression and publication bias were assessed. Individual participant data were used to estimate effects by logistic regression and to evaluate BNP/NT-proBNP additional predictive value by area under the receiver operating characteristic curves, and integrated discrimination improvement and categorical net reclassification improvement indexes.

Results: Literature-based meta-analysis included 3,498 stroke patients from 16 studies and revealed that BNP/NT-proBNP levels were $255.78 \mathrm{pg} / \mathrm{mL}$ (95\% confidence interval [CI] 105.10-406.47, $p=0.001$ ) higher in patients who died; publication bias entailed the loss of this association. Individual participant data analysis comprised 2,258 stroke patients. After normalization of the data, patients in the highest quartile had double the risk of death after adjustment for clinical variables (NIH Stroke Scale score, age, sex) (odds ratio 2.30, 95\% Cl 1.32-4.01 for BNP; and odds ratio 2.63, 95\% Cl 1.75-3.94 for NT-proBNP). Only NT-proBNP showed a slight added value to clinical prognostic variables, increasing discrimination by 0.028 points (integrated discrimination improvement index; $p<0.001$ ) and reclassifying $8.1 \%$ of patients into correct risk mortality categories (net reclassification improvement index; $p=0.003$ ). Neither etiology nor time from onset to death affected the association of BNP/NT-proBNP with mortality.

Conclusion: BNPs are associated with poststroke mortality independent of NIH Stroke Scale score, age, and sex. However, their translation to clinical practice seems difficult because BNP/NT-proBNP add only minor predictive value to clinical information. Neurology ${ }^{\circledR}$ 2013;81:1976-1985

\section{GLOSSARY}

AUC $=$ area under the receiver operating characteristic curve; BNP = B-type natriuretic peptide; $\mathbf{C l}=$ confidence interval; IDI = integrated discrimination improvement; IPD = individual participant data; IQR = interquartile range; $\mathbf{N I H S S}=\mathrm{NIH}$ Stroke Scale; $\mathbf{N R I}$ = net reclassification improvement; $\mathbf{N T}$-proBNP = N-terminal fragment of B-type natriuretic peptide; $\mathbf{O R}=$ odds ratio; WMD = weighted mean difference.

Early prediction of fatal outcome after stroke might improve decision-making processes, such as the use of more aggressive therapies or the inclusion of selected patients in clinical trials. Clinical models to predict death after stroke perform with reasonable accuracy, ${ }^{1,2}$ but the addition of blood biomarkers might lead to better predictive models. ${ }^{3,4}$

B-type natriuretic peptide (BNP), a vasoactive hormone with natriuretic, diuretic, and vasodilator activity, is synthesized mainly in cardiac tissue as a result of myocyte stretch. ${ }^{5}$ Activation of BNP generates an inactive N-terminal fragment (NT-proBNP) ${ }^{6}$ and both peptides have been associated with unfavorable clinical outcome and mortality in stroke patients. ${ }^{7-9}$ Two recent

\footnotetext{
From the Neurovascular Research Laboratory (T.G.-B., D.G., A.B., J.M.), Vall d'Hebron Institute of Research, Universitat Autònoma de Barcelona, Spain; Department of Neurology (T.E.), Kliniken Südostbayern-Klinikum Traunstein, Germany; Department of Psychiatry and Psychotherapy (T.E.), Technische Universität München, Germany; Department of Cardiology (J.K.J.), Odense University, Denmark; Stroke Medicine (J.C.S.), Lincoln County Hospital, University of Nottingham, UK; Department of Stroke Medicine (K.S.), Kawasaki Medical School, Kurashiki City, Okayama, Japan; Department of Emergency Medicine (A.S.), School of Medicine, Duzce University, Turkey; Department of Neurology (X.C.), Fujian Provincial Hospital, Fujian Medical University, Fuzhou, China; and Centre for Clinical Brain Sciences (W.N.W.), University of Edinburgh, UK. Go to Neurology.org for full disclosures. Funding information and disclosures deemed relevant by the authors, if any, are provided at the end of the article.
} 
studies $^{10,11}$ show that, although BNP has a statistically significant association with mortality and functional outcome, it does not add prognostic information to simple clinical variables. However, this new information should be carefully managed, because both studies were small and BNP-related peptides are important predictors of poor outcome in other diseases. ${ }^{12,13}$

Because there is still uncertainty about whether BNP could be useful in stroke practice to predict mortality, first we conducted a systematic review and a literature-based meta-analysis to examine the association of BNP/NT-proBNP levels with all-cause mortality after a cerebrovascular event. Second, we performed an individual participant data (IPD) analysis to further explore the role of natriuretic peptides and to assess whether their addition to clinical information could have additional predictive value and/or could improve mortality risk stratification.

METHODS Standard protocol approvals, registrations, and patient consents. Methods for this study were specified in advance and registered in the PROSPERO database (CRD42012003284). ${ }^{14}$ We report it in reference to the PRISMA (Preferred Reporting Items for Systematic Reviews and Meta-Analyses) statement. ${ }^{15}$

Eligibility criteria and search strategy. We included studies that reported original data and i) recruited patients with ischemic stroke, TIA, and/or hemorrhagic stroke; ii) measured BNP or NT-proBNP; and iii) recorded whether patients died during follow-up.

We searched MEDLINE and EMBASE databases up to October 26, 2012 without restriction (appendix e-1 on the Neurology ${ }^{\circledR}$ Web site at www.neurology.org). Reference lists from identified articles and published reviews were hand-searched.

Two independent reviewers thoroughly checked titles and/or abstracts yielded by search and also screened potentially relevant articles in full text after removing duplicates. Disagreements were resolved by discussion.

Data collection. Two independent reviewers extracted data using a simple, standardized template. Appendix e-2 shows items included in that template. If data were missing, the corresponding author was contacted by e-mail.

Three independent reviewers assessed the quality of each included article using a 15-point quality score (appendix e-3), which does not apply for inclusion of the article in the meta-analysis. Corresponding authors from included articles were invited to participate sharing individual records using a preplanned dataset including baseline variables for IPD analysis (see appendix e-4).

Statistical analysis. STATA 10.0 (StataCorp, College Station, TX) and SPSS 15.0 (SPSS Inc., Chicago, IL) were used for statistical analyses, unless otherwise stated.

Literature-based meta-analysis. The strength of association of BNP/NT-proBNP with death was measured by weighted mean differences (WMDs) between death and survival groups with a random effects model. Cochrane $Q$ and $I^{2}$ statistics measured heterogeneity. WMD not including zero on its confidence interval (CI) and with a $t$ test $p$ value $<0.05$ were considered significant; forest plot summarized these size effects. We used meta-regression to adjust our study level meta-analysis for the potential confounders. We used Duval-Tweedie nonparametric "trim-and-fill" test ${ }^{16}$ to assess publication bias.

IPD analysis. A whole compiled database including all individual information was used as a unique cohort to perform a complete statistical analysis. We assessed publication bias with Duval-Tweedie test for both BNP and NT-proBNP separately. BNP and NT-proBNP levels had a nonnormal distribution (KolmogorovSmirnov test with $p<0.05$ ), so we applied Mann-Whitney $U$ or Kruskal-Wallis tests and reported median and interquartile range (IQR). Pearson $\chi^{2}$ test assessed differences among categorical variables. Correlations with stroke severity measured by NIH Stroke Scale (NIHSS) at admission and age were performed by Spearman rank.

Data from all different cohorts were normalized by standardized $z$ scores in the unique database. We constructed forward stepwise multivariate logistic regression models with all clinical variables associated with mortality at $p<0.05$ (baseline NIHSS score, age, and sex). Odds ratio (OR), $95 \% \mathrm{CI}$, and $p$ value were given. Biomarker levels, using the highest quartile cutoff point, were added to the logistic regression model including clinical variables. To gain results consistency, we performed bootstrap calculation for OR and $95 \% \mathrm{CI}$ in a random sample with replacement of the total number of patients, using a modified version of Car R-package. ${ }^{17}$

The method of DeLong et al. ${ }^{18}$ allowed us to compare the areas under the receiver operating characteristic curve (AUCs) from models including biomarkers with AUCs from clinical model only using MedCalc 12.3 software. Using R software (Hmisc and PredictABEL packages), net reclassification improvement (NRI) and integrated discrimination improvement (IDI) indexes assessed the added value of the biomarker to the clinical model to predict mortality. ${ }^{19}$ For the NRI test, prespecified clinically relevant thresholds of predicted risk $(<10 \%$ and $>90 \%)$ were used to calculate reclassification of patients.

In all cases, a $p$ value $<0.05$ was considered significant at a 95\% confidence level. For multitesting, we applied Bonferroni correction.

RESULTS Study selection and characteristics. Our search strategy yielded 957 references, 20 of which were considered for full screening. Finally, 16 articles met inclusion criteria and were included in the meta-analysis (figure 1).

Table 1 presents the characteristics of included studies..$^{7-11,20-30}$ The quality of the 16 included articles was moderate (7 points, IQR 9-11; minimum 5, maximum 13). Only $25 \%$ of articles specified that measurement of biomarker was blind to clinical data; only one article reported sample size calculation (table e-1).

Synthesis of results from literature-based meta-analysis. The included articles recruited a total of 3,498 stroke patients. Although BNP/NT-proBNP mean levels were higher in nonsurvivors than survivors, all studies showed large deviations (table 1) and a high heterogeneity between them $\left(Q=274.92, I^{2}=94.5 \%\right.$, $d f 15, p<0.001)$.

On average, BNP/NT-proBNP levels were $255.78 \mathrm{pg} / \mathrm{mL}$ higher in those patients who died 
Figure 1 Flow diagram

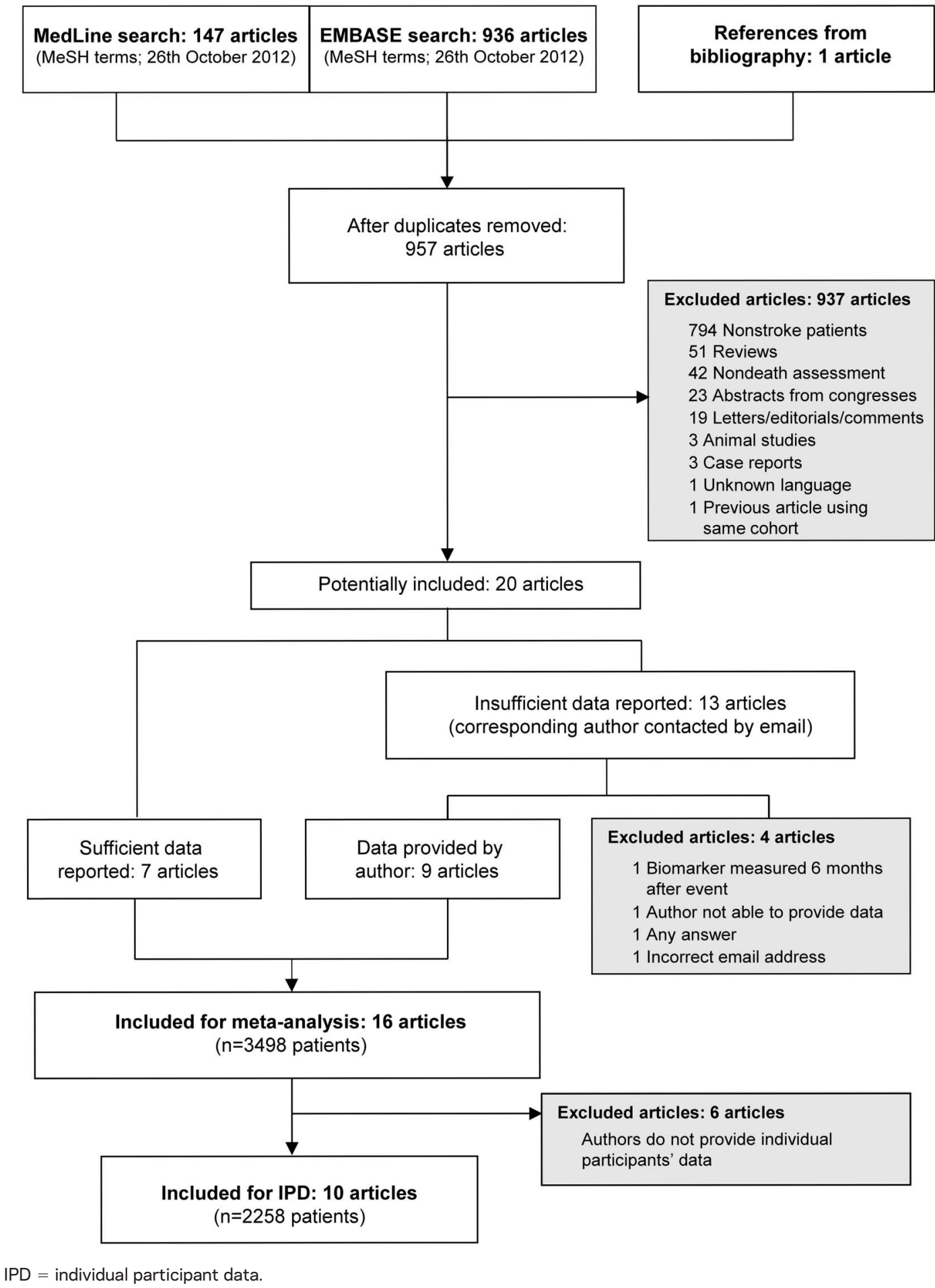

(95\% CI 105.10-406.47, $p=0.001$ ) (WMDs for each individual study and all-studies average are given in figure 2). This association was not affected by any of the study level confounders that were considered, including BNP vs NT-proBNP, stroke severity, age, plasma or serum measurement, time of sample collection, sex, and quality score. However, there exists uncertainty about the value of this result because study bias was detected by loss of significance when the Duval-Tweedie test was applied (pooled WMD $121.55 \mathrm{pg} / \mathrm{mL}$, 95\% $\mathrm{CI}-33.56$ to $276.66, p=0.125)$.

Synthesis of results from IPD analysis. We obtained individual information from 2,258 stroke patients from 10 included articles. ${ }^{8-11,20,21,24,25,27,30}$ In the compiled dataset, we found NT-proBNP levels almost 10 


\begin{tabular}{|c|c|c|c|c|c|c|c|c|c|c|c|}
\hline Ref. & Marker & Method & Blood collection & Sample size & Time of death & Death, \% & NIHSS adm. & Age, y & Males, \% & $\begin{array}{l}\text { Biomarker in deceased } \\
\text { patients }\end{array}$ & $\begin{array}{l}\text { Biomarker in alive } \\
\text { patients }\end{array}$ \\
\hline 20 & 2 & Elecsys2010 & $<12 \mathrm{~h}$ & 174 & $90 \mathrm{~d}$ & 10.9 & $9.8 \pm 7.9$ & $67.7 \pm 11.6$ & 59.2 & $4,053.8 \pm 6,138.3$ & $1,009.4 \pm 1,741.6$ \\
\hline 7 & 2 & Radioimmunoassay & $2 d$ & 51 & $4 y$ & 43.1 & $12.9 \pm 19.8$ & $67 \pm 10$ & 43.1 & $11,909.1 \pm 19,352.1$ & $2,070.3 \pm 2,083.0$ \\
\hline 8 & 2 & Elecsys2010 & Adm. & 250 & $6 \mathrm{mo}$ & 9 & $9 \pm 7.9$ & $68.6 \pm 12.8$ & 53.2 & $1,491.1 \pm 1,847.5$ & $825.9 \pm 2,692.5$ \\
\hline 21 & 2 & Modular Analytics E170 & $5 \mathrm{~d}$ (mean) & $114^{a}$ & $120 d$ & 11.4 & $11.2 \pm 5.9$ & $72.9 \pm 12.6$ & 48.2 & $8,956.0 \pm 11,097.3$ & $1,060.3 \pm 2,070.6$ \\
\hline 22 & 2 & - & $48 \mathrm{~h}$ (median) & $72^{\mathrm{a}}$ & In-hospital & 25 & - & $63 \pm 13$ & 40 & $11,898 \pm 12,741$ & $4,073 \pm 5,691$ \\
\hline 23 & 2 & Modular Analytics E170 & $<5 \mathrm{~h}$ & $41^{\mathrm{a}}$ & In-hospital & 12.2 & $7.5 \pm 6.2$ & $78.3 \pm 6.8$ & 36 & $950.1 \pm 1,567.1$ & $873.2 \pm 1,415.7$ \\
\hline 9 & 1 & Immunoassay (Shionogi) & $<24 \mathrm{~h}$ & 335 & In-hospital (1 mo) & 6 & $9 \pm 8.1$ & $72.3 \pm 12.3$ & 62.7 & $731.5 \pm 1,070.9$ & $213.1 \pm 384.5$ \\
\hline 24 & 2 & Modular Analytics E170 & $3 \mathrm{~d}$ (mean) & $125^{a}$ & 1 y & 16 & $13.4 \pm 20.1$ & $73 \pm 12$ & 49.6 & $8,289.5 \pm 10,570.4$ & $1,060.5 \pm 2,071.1$ \\
\hline 25 & 1 & Triage (Biosite) & $<12 \mathrm{~h}$ & 60 & In-hospital & 26.7 & $10.6 \pm 6.3$ & $60.7 \pm 14.1$ & 41.2 & $172.2 \pm 226.4$ & $111.0 \pm 146.6$ \\
\hline 26 & 2 & Immulite 2500 (Siemens) & Adm. & $92^{\mathrm{a}}$ & In-hospital & 30.4 & - & $65.6 \pm 11$ & 44.6 & $1.8 \pm 0.9$ & $0.7 \pm 0.8$ \\
\hline 27 & 1 & Immunoassay (Shionogi) & $<24 \mathrm{~h}$ & $221^{a}$ & In-hospital & 10.9 & $12.1 \pm 8.9$ & $76.5 \pm 10.9$ & 54.7 & $714.1 \pm 716.3$ & $320.0 \pm 380.7$ \\
\hline 10 & 2 & Elecsys2010 & $<24 \mathrm{~h}$ & $270^{a}$ & $90 \mathrm{~d}$ & 11 & $6 \pm 7.0$ & $74.4 \pm 12.5$ & 41.8 & $6,852.6 \pm 7,541.0$ & $1,152.4 \pm 1,777.9$ \\
\hline 28 & 2 & Immunoassay (Biomedica Gruppe) & $<48 \mathrm{~h}$ & 569 & $6 \mathrm{mo}$ & 5.8 & - & $67.9 \pm 15$ & 54 & $379.6 \pm 200.4$ & $251.5 \pm 167.1$ \\
\hline 29 & 2 & COBAS h232 (Roche) & Adm. & 106 & In-hospital & 21.7 & $15.5 \pm 6.5$ & $69.2 \pm 11.9$ & 58.5 & $508.3 \pm 121.3$ & $153.4 \pm 63.4$ \\
\hline 11 & 1 & Immunoassay (Biosite) & $<24 \mathrm{~h}$ & $896^{a}$ & In-hospital & 9.3 & $8.2 \pm 7.0$ & $72 \pm 12$ & 52 & $186.1 \pm 282.3$ & $123.8 \pm 219.7$ \\
\hline 30 & 2 & - & $<72 \mathrm{~h}$ & 122 & In-hospital & 18.8 & $9.8 \pm 5.1$ & $71.5 \pm 9.8$ & 56.6 & $4,642.3 \pm 4,097.5$ & $1,626.0 \pm 2,370.4$ \\
\hline
\end{tabular}

Abbreviations: Adm. = at admission; BNP = B-type natriuretic peptide; NIHSS = NIH Stroke Scale; NT-proBNP = N-terminal fragment of BNP; Ref. $=$ reference.

Data represent mean \pm SD for NIHSS score, age, and biomarker levels. Biomarker levels given as pg/mL. Marker: $2=$ NT-proBNP; $1=$ BNP. $-=$ information not available.

a Study including more than one type of event (i.e., ischemic and hemorrhagic and/or TIA). 


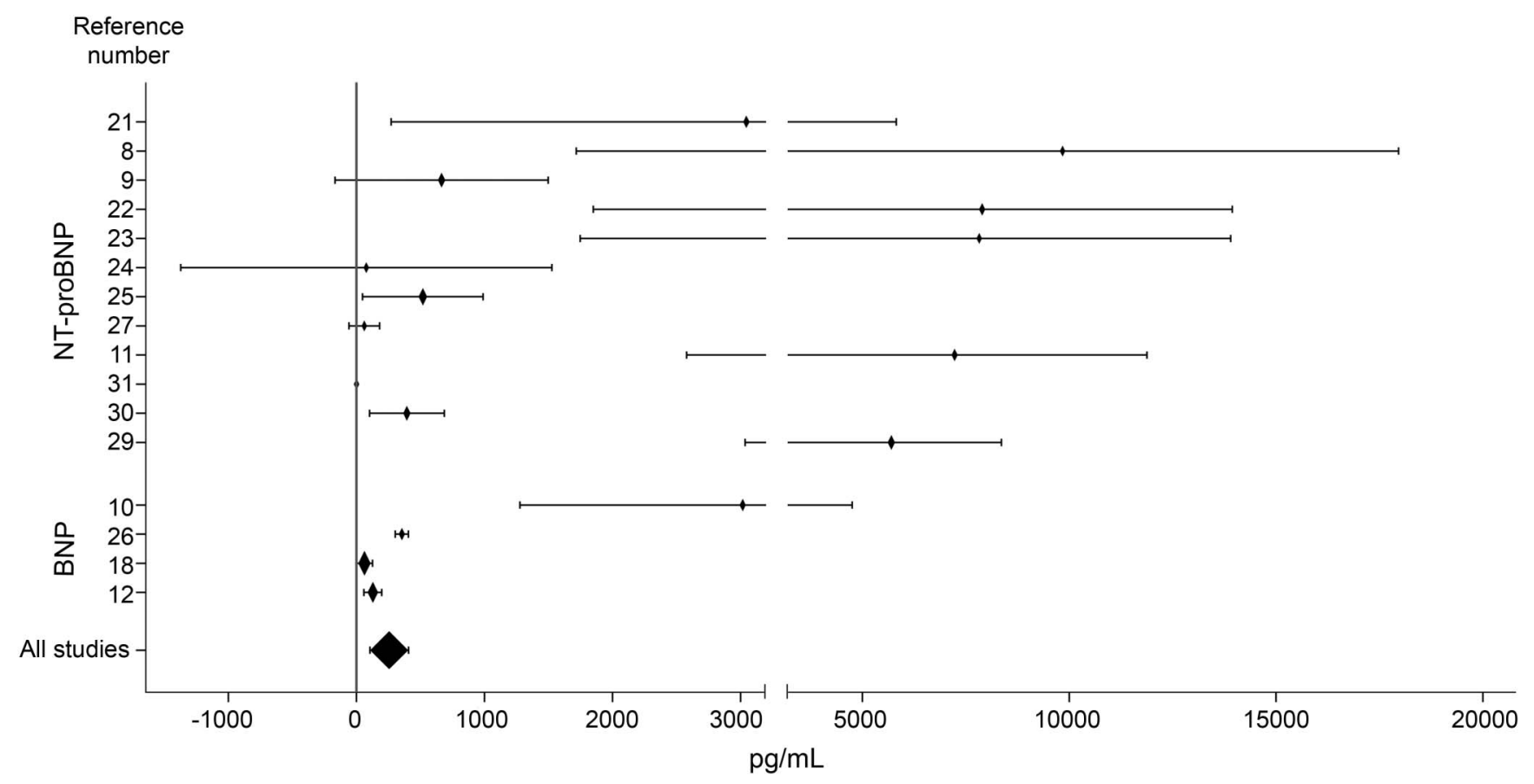

Weighted mean differences (diamonds) for B-type natriuretic peptide (BNP) and N-terminal fragment of BNP (NT-proBNP) levels between death and survival groups. Lines represent $95 \%$ confidence interval. Sizes of diamonds are proportional to sample size.

times higher than BNP levels (401 pg/mL [IQR 1261,375 ] vs $77.8 \mathrm{pg} / \mathrm{mL}$ [IQR 27.8-191]; $p<0.001$ ). Therefore, analyses for the 2 peptides were performed separately. Neither BNP nor NT-proBNP changed the degree of association when the Duval-Tweedie test was applied. There was therefore no evidence of publication bias (data not shown). However, for BNP (with only 3 studies), the association with mortality did not reach statistical significance when WMDs were considered (pooled WMD $113.75 \mathrm{pg} / \mathrm{mL}, p=0.164$ ).

We assessed the association of BNP and NTproBNP levels with several demographic and clinical characteristics. Higher levels of both peptides were found in women (119 [43-244.9] vs 59.9 [19.4$180.4] \mathrm{pg} / \mathrm{mL}, p<0.001$ for BNP; and 414.4 [139.5-1,822.5] vs 279.1 [59.2-1463.1] $\mathrm{pg} / \mathrm{mL}$, $p<0.001$ for NT-proBNP), nonsmokers (89.4 [32-216.2] vs $54.4[17.2-192.5] \mathrm{pg} / \mathrm{mL}, p=0.001$ for BNP; $507.4[109.9-1,911.3]$ vs 279.1 [84.6$820.3] \mathrm{pg} / \mathrm{mL}, p<0.001$ for NT-proBNP), and patients with atrial fibrillation (207.6 [108-356] vs 44.6 [14.6-113.3] $\mathrm{pg} / \mathrm{mL}, p<0.001$ for BNP; $1,864.8$ [816.1-4368] vs 164.9 [59.2-524.3] $\mathrm{pg} / \mathrm{mL}, p<$ 0.001 for NT-proBNP). Both BNP and NT-proBNP levels positively correlated with NIHSS score at admission $(r=0.228, p<0.001$ for BNP; $r=0.272, p<$ 0.001 for NT-proBNP) and age $(r=0.340, p<$ $0.001 ; r=0.477, p<0.001$, respectively).

Because our systematic search was focused only on ischemic events, we further analyzed only ischemic stroke patients regarding mortality, and after exclusion of patients with hemorrhagic stroke or TIA, the sample size was 957 patients with BNP measurement and 880 patients for NT-proBNP. Table 2 presents demographic characteristics for each individual study and for pooled data. After data normalization, we divided patients into quartiles for both BNP and NT-proBNP and when the highest quartile was compared with the others, BNP was independently associated with poststroke mortality, giving a pooled adjusted OR of 2.30 (95\% CI 1.32-4.01, $p=0.003)$ after logistic regression adjustment for NIHSS score, age, and sex. NT-proBNP was also associated with mortality after stroke (adjusted $\mathrm{OR}=2.63$, $95 \%$ CI 1.75-3.94, $p<0.001)$. These results indicate a double risk of death for those patients in the highest quartile for both peptides.

By using these cutoff points, only NT-proBNP showed a slight added value to model with clinical variables alone (NIHSS score, age, sex), showing an increase in AUC $(p=0.029)$. Regarding discrimination improvement measured with the IDI index, both markers showed a small increase $(0.018$ points for BNP and 0.028 points for NT-proBNP). However, when reclassification of patients into predefined categories of risk of death was analyzed, again only the addition of NT-proBNP to simple clinical variables showed added value, classifying $8.1 \%$ of patients (NRI index; $p=$ 0.003 ) into more accurate risk categories than a model based on clinical variables alone (table e- 2 and graphical representation in figure 3). 


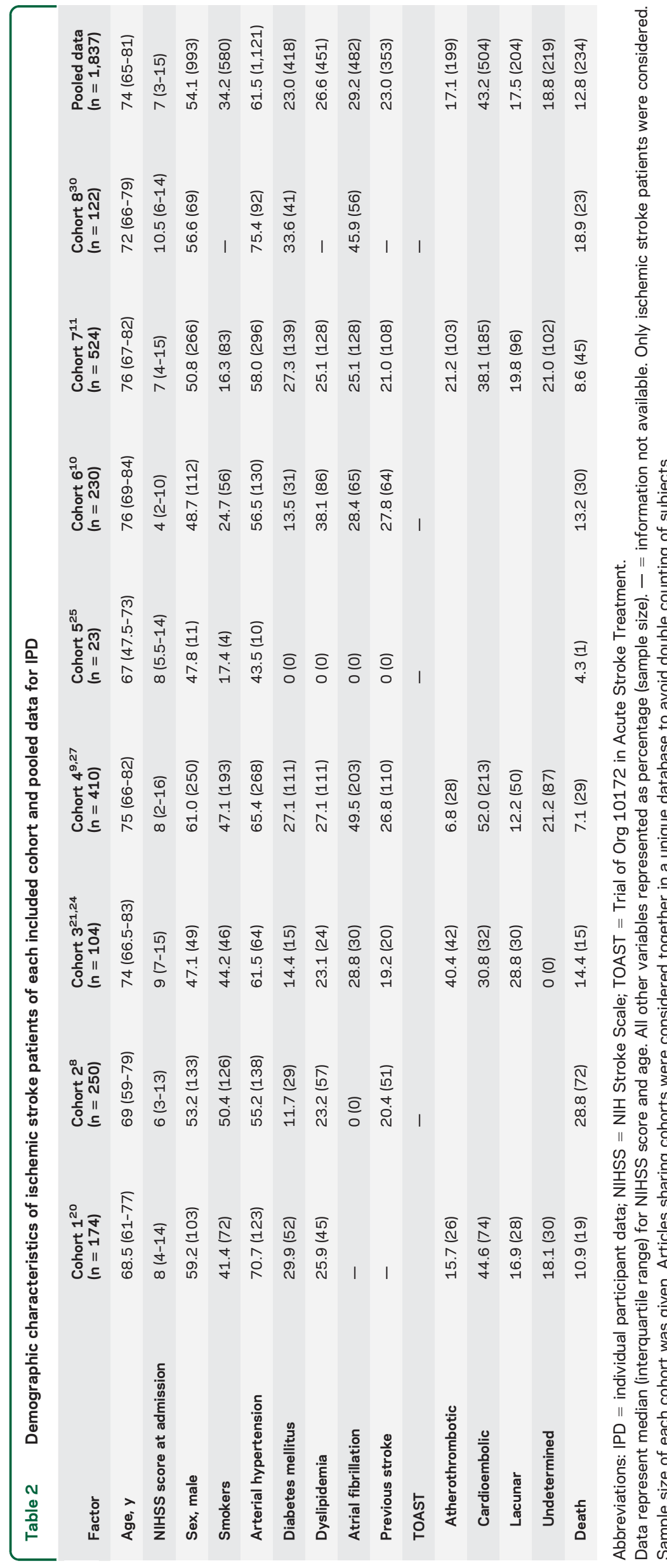

Furthermore, for those patients who died, we explored BNP/NT-proBNP blood levels regarding the time that passed from stroke to death and no statistical difference was found (data not shown). The association with mortality was subanalyzed considering ischemic stroke etiologies. BNP/NT-proBNP levels were higher in cardioembolic strokes but only when alive patients were considered; we found differences in circulating levels between dead and alive patients in cardioembolic etiology, but also in lacunar (for BNP) and atherothrombotic (for NT-proBNP) strokes (figure e-1). Time of sample collection did not affect the association of BNP or NT-proBNP level with mortality (figure e-2).

We found similar results when nonnormalized data were used, for both predictive models (in which the highest quartile corresponds to BNP $>216.40$ $\mathrm{pg} / \mathrm{mL}$ and NT-proBNP $>1,453.45 \mathrm{pg} / \mathrm{mL}$ ) and subanalyses (data not shown).

DISCUSSION Our meta-analyses confirmed the association of BNPs with mortality after stroke, independent of age, sex, and baseline neurologic impairment. However, although this association was statistically significant, these biomarkers did not lead to better prediction of death than clinical information alone.

The design used in this study has allowed us to perform a thorough analysis. Our literature-based metaanalysis performed after systematic review, with close to 3,500 patients, gave the statistical power needed to estimate whether a real association exists between BNP/NT-proBNP and death. Our results confirmed the presence of higher circulating BNP/NT-proBNP levels in those patients who died after a cerebrovascular event. ${ }^{8,9,30}$ However, we found moderate quality of reporting (7 of 15 points), high heterogeneity among included studies (94.5\%), and evidence of publication bias. Given this, the results of the literature-based meta-analysis should be carefully interpreted. Publication of negative studies has generally less acceptation and impact, and perhaps the lack of published negative studies in the field of biomarkers affects the results of meta-analyses; the publication of negative results may contribute first to give a more realistic point of view and second to save resources for other researchers.

A strong point in our design was the IPD analysis. We considered several covariates at patient level, being the least biased and most reliable means of addressing questions not resolved in independent studies. ${ }^{31} \mathrm{We}$ performed IPD analysis following a one-stage approach $^{32}$ (using the compiled database as a unique cohort). The IPD analysis showed both BNP and NT-proBNP as independent predictors of mortality after stroke, even after adjustment by typical confounders (such as NIHSS score, sex, and age). To have $\mathrm{BNP} / \mathrm{NT}$-proBNP levels in the highest quartile 
A

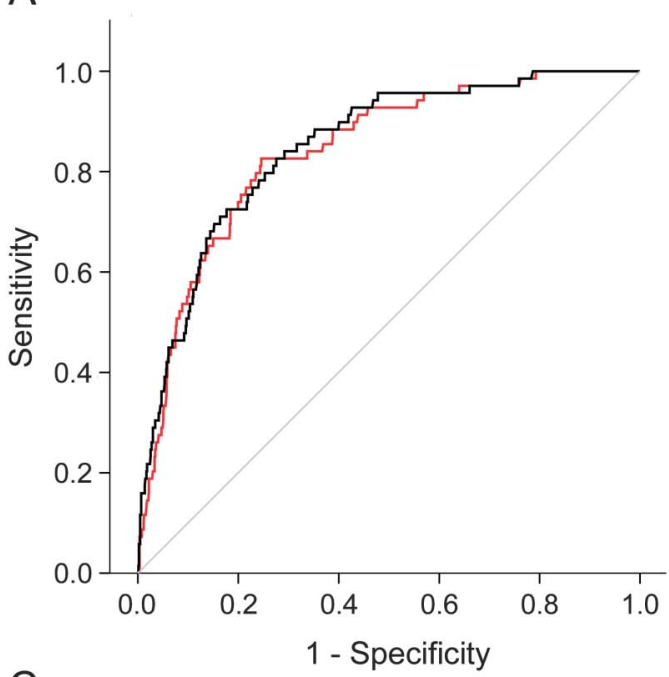

C

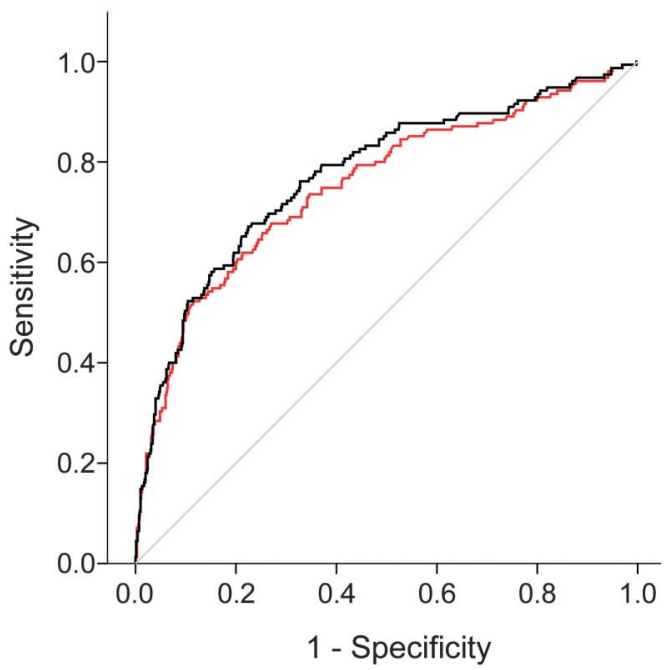

B

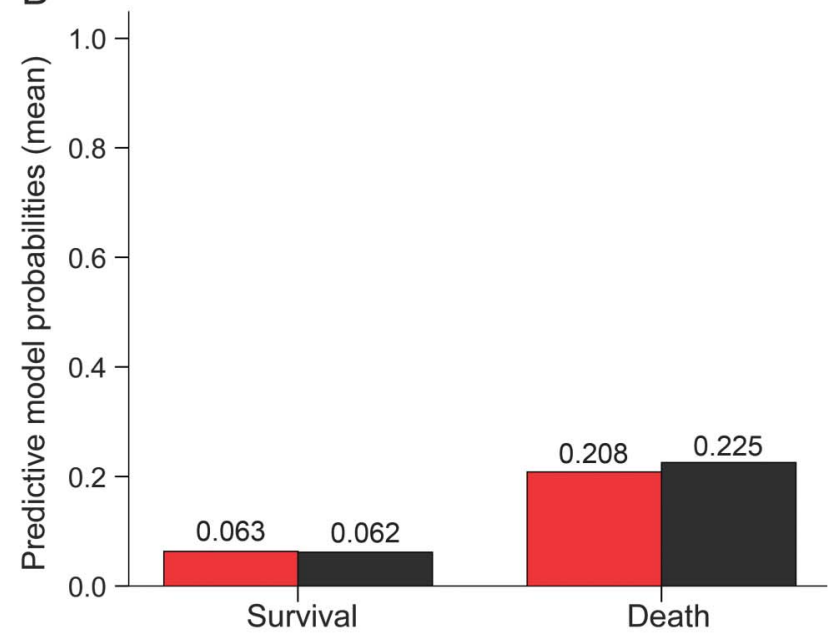

D

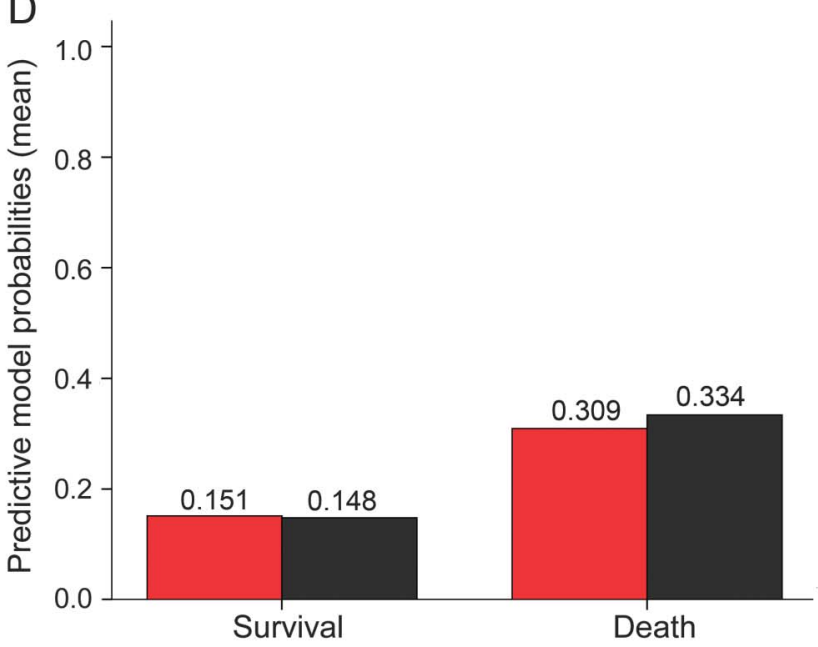

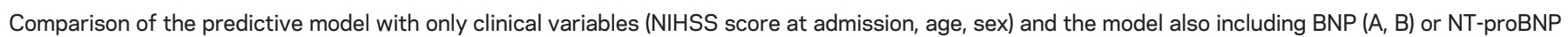

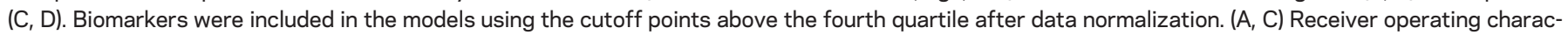

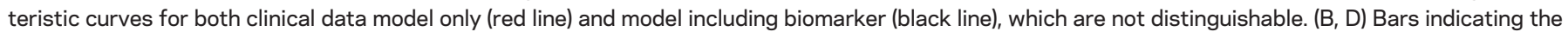

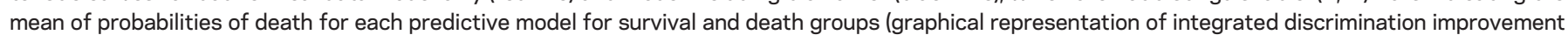

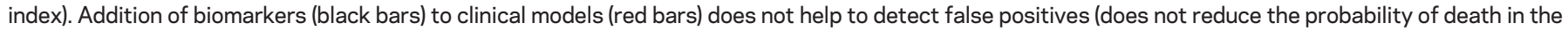

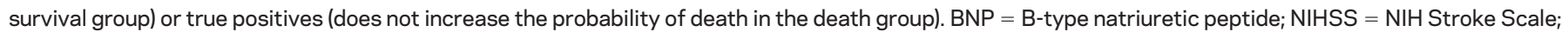
$\mathrm{NT}$-proBNP $=\mathrm{N}$-terminal fragment of BNP.

doubled the risk of death after ischemic stroke compared with other quartiles.

Furthermore, one-stage IPD allows the performance of more complete statistical analysis in large cohorts (more than 2,000 patients in our case). We detected differences between BNP and NT-proBNP levels: NT-proBNP showed almost 10-fold higher concentration. This contrast might be attributable mainly to differences in the metabolism of these 2 peptides, which implies different half-lives, the longer corresponding to NT-proBNP, ${ }^{33}$ and thus it justifies the analysis of each molecule separately.

Our main interest was to assess whether these biomarkers could improve the information given by clinical information alone by applying different statistical metrics, such as IDI and NRI. ${ }^{19}$ In our IPD analysis, only NT-proBNP showed a marginal additional value over clinical data. Based on these results, we would not recommend the use of BNPs alone at the bedside to predict stroke outcome. Because currently there is not consensus about how much a marker must add to clinical models in terms of discrimination and reclassification improvements, with our results, only NT-proBNP might be considered in a future panel of prognostic biomarkers, including other known (interleukin-6, copeptin, etc.) or still unknown blood outcome biomarkers, in which each biomarker potentially increases discrimination and reclassification, reaching outstanding accuracy. 
Recently, the possibility has been suggested that the prognostic value of natriuretic peptides is dependent on stroke etiology. ${ }^{28}$ We detected higher levels of both BNP and NT-proBNP in cardioembolic strokes only when alive patients were considered; moreover, circulating BNP and NT-proBNP were not exclusively associated with mortality in cardioembolic, but also in other causes of stroke. In addition, recently, high levels of BNP have been associated with cardioembolic etiology and with infarct volume in stroke patients, ${ }^{34}$ reinforcing the heart-brain link and the suggestion that cardiovascular factors, in particular cardiac failure, adversely influence acute stroke outcome. ${ }^{35}$ In our meta-analyses, we have focused on the relationship between BNP/NT-proBNP and stroke mortality and their role as useful biomarkers; therefore, the potential role of BNP/NT-proBNP in the detection of cardioembolic etiology, ${ }^{36}$ mainly to define cryptogenic strokes, ${ }^{37}$ needs further exploration and we suggest following a similar approach to the one presented here.

We believe that the implementation of these types of statistical analyses together with the proposal of minimal quality request (like other guidelines stated for publication ${ }^{16}$ ) in biomarker studies would minimize the risk of bias and facilitate translation into clinical

\section{Comment: \\ Natriuretic peptides as predictive biomarkers of stroke outcome}

B-type natriuretic peptide (BNP), along with an inactive $\mathrm{N}$-terminal peptide fragment (NT-proBNP), is secreted by cardiac ventricular myocytes in response to excessive myocardial stretching. The plasma half-life of these peptides is 0.3 and 2.0 hours, respectively, so a single measurement mostly reflects recent cardiac stress that may, for example, reflect sympathetic stimulation in response to acute stroke. Results from a variety of clinical studies indicate that these peptides may be useful as biomarkers for a variety of both cardiac and cerebrovascular events. In a carefully performed meta-analysis, García-Berrocoso and colleagues ${ }^{1}$ evaluate the relationship of BNP and NT-proBNP levels with mortality following acute stroke. Although individuals in the highest quartile for either of the 2 peptides had twice the risk of death compared to the lower quartiles, only the NT-proBNP measures added slightly (additional $8.1 \%$ of patients) to the typical clinical measures for predicting stroke mortality, including age, sex, and NIH Stroke Scale score. The results do not go far enough to establish a relationship between the cause of death and the highest natriuretic levels. Some suggestive data from the ARISTOTLE trial indicate that cardiac death has an important role, but more careful studies that focus on the usefulness of measuring BNP or NT-proBNP within the first 24 hours after acute stroke, along with an analysis of the explicit causes of death, are needed. ${ }^{2}$

1. García-Berrocoso T, Giralt D, Bustamante A, et al. B-type natriuretic peptides and mortality after stroke: a systematic review and meta-analysis. Neurology 2013;81: $1976-1985$.

2. Hijazi Z, Wallertin L, Siegbahn A, et al. N-terminal pro-B-type natriuretic peptide for risk assessment in patients with atrial fibrillation: insights from the ARISTOTLE Trial (Apixaban for the Prevention of Stroke in Subjects with Atrial Fibrillation). J Am Coll Cardiol 2013;61:2274-2284.

Bruce M. Coull, MD

From the University of Arizona Health Science Center, Tucson.

Study funding: No targeted funding reported.

Disclosure: B. Coull serves/has served on scientific advisory boards for NIH, NINDS, Astra Zeneca, Neurobiological Technologies, and Boehringer-Ingelheim; and receives research support from NIH/NINDS. Go to Neurology.org for full disclosures. practice. Finding a way to predict poor outcome in those patients with stroke would provide information for patients and relatives, would help to evaluate the risk and benefits of acute treatments or inclusion into clinical trials, and would optimize the allocation into specialized stroke units.

Our meta-analysis presents some limitations. First, there were differences in methodology among the included articles, for both the method of analysis and time of blood collection, which could influence our results. Second, our systematic review focused on ischemic stroke, thus studies assessing mortality in hemorrhagic stroke or TIA were missed. Nevertheless, some of the included articles considered a mixed population, with ischemic as well as hemorrhagic strokes and/or TIA, which could not be separated in our literaturebased meta-analysis but were excluded in the IPD. Third, for subanalysis of mortality by stroke etiology, our conclusions should be carefully interpreted because of limited sample size: stroke etiology classification by TOAST (Trial of Org 10172 in Acute Stroke Treatment) was available in only half of the patients in the IPD analysis, including 278 patients with NT-proBNP measurement and 934 for BNP.

Finally, our IPD analysis, although considering several covariates, does not include some pathologies related to increased levels of BNP/NT-proBNP such as cardiopathies different from atrial fibrillation ${ }^{38}$ or renal disease. ${ }^{39}$ In addition, we could not access data regarding specific causes of death after stroke, which could influence the level of association of BNP/NT-proBNP.

In conclusion, both our literature-based meta-analysis and IPD analysis reinforce the role of BNP/NT-proBNP as independent predictors of all-cause mortality after stroke. However, these natriuretic peptides add minor predictive value to clinical information for the prediction of death. Their role in the clinical identification of an ischemic stroke of cardioembolic origin is uncertain and needs more research.

\section{AUTHOR CONTRIBUTIONS}

Study concept and design (T.G.-B., D.G., W.N.W., J.M.), analysis and interpretation of data (T.G.-B., D.G., A.B.), drafting and revising the manuscript (T.G.-B., D.G., A.B., T.E., J.K.J., J.C.S., K.S., A.S., W.N.W., X.C., J.M.), acquisition of data (T.E., J.K.J., J.C.S., K.S., A.S., W.N.W., X.C., J.M.), statistical analyses (T.G.-B., D.G.), study supervision or coordination (T.G.-B., J.M.). T.G.-B. and J.M. have full access to all of the data and take full responsibility for the data, the analyses, and interpretation. All authors reviewed and approved the final report.

\section{ACKNOWLEDGMENT}

The authors are grateful to Natalia Rost, MD, MPH (Boston, MA), Emina Hajdinjak, MD (Maribor, Slovenia), and Pedro J. Modrego (Zaragoza, Spain) for their kind comments during protocol preparation.

\section{STUDY FUNDING}

No targeted funding reported. 


\section{DISCLOSURE}

T. García-Berrocoso is supported by a predoctoral fellowship (FI09/ 00017) from the Instituto de Salud Carlos III. Neurovascular Research Laboratory takes part in the Spanish stroke research network INVICTUS (RD12/0014/0005) and is supported on stroke biomarkers research by FIS 11/0176. D. Giralt, A. Bustamante, T. Etgen, J. Jensen, J. Sharma, K. Shibazaki, A. Saritas, and X. Chen report no disclosures. W. Whiteley was supported by the Chief Scientist's Office (CAF/06/30) and is now funded by a UK Medical Research Council Clinician Scientist Fellowship (G0902303). J. Montaner reports no disclosures. Go to Neurology.org for full disclosures.

Received March 21, 2013. Accepted in final form July 29, 2013.

\section{REFERENCES}

1. Saposnik G, Kapral MK, Liu Y, et al. IScore: a risk score to predict death early after hospitalization for an acute ischemic stroke. Circulation 2011;123:739-749.

2. O'Donnell MJ, Fang J, D'Uva C, et al. The PLAN Score: a bedside prediction rule for death and severe disability following acute ischemic stroke. Arch Intern Med 2012; 172:1548-1556.

3. Whiteley W, Chong WL, Sengupta A, Sandercock P. Blood markers for the prognosis of ischemic stroke: a systematic review. Stroke 2009;40:e380-e389.

4. Whiteley W, Jackson C, Lewis S, et al. Inflammatory markers and poor outcome after stroke: a prospective cohort study and systematic review of interleukin-6. PLoS Med 2009;6:e1000145.

5. deLemos JA, McGuire DK, Drazner MH. B-type natriuretic peptide in cardiovascular disease. Lancet 2003;362:316-322.

6. Levin ER, Gardner DG, Samson WK. Natriuretic peptides. N Engl J Med 1998;339:321-328.

7. Mäkikallio AM, Mäkikallio TH, Korpelainen JT, et al. Natriuretic peptides and mortality after stroke. Stroke 2005;36:1016-1020.

8. Jensen JK, Mickley H, Bak S, Korsholm L, Kristensen SR. Serial measurements of $\mathrm{N}$-terminal pro-brain natriuretic peptide after acute ischemic stroke. Cerebrovasc Dis 2006;22:439-444.

9. Shibazaki K, Kimura K, Okada Y, et al. Plasma brain natriuretic peptide as an independent predictor of in-hospital mortality after acute ischemic stroke. Intern Med 2009;48:1601-1606.

10. Whiteley W, Wardlaw J, Dennis M, et al. The use of blood biomarkers to predict poor outcome after acute transient ischemic attack or ischemic stroke. Stroke 2012;43:86-91.

11. Montaner J, García-Berrocoso T, Mendioroz $\mathrm{M}$, et al. Brain natriuretic peptide is associated with worsening and mortality in acute stroke patients but adds no prognostic value to clinical predictors of outcome. Cerebrovasc Dis 2012;34:240-245.

12. Januzzi JL Jr, Maisel AS, Silver M, Xue Y, DeFilippi C. Natriuretic peptide testing for predicting adverse events following heart failure hospitalization. Congest Heart Fail 2012;18:S9-S13.

13. Coutance G, Le Page O, Lo T, Hamon M. Prognostic value of brain natriuretic peptide in acute pulmonary embolism. Crit Care 2008;12:R109.

14. Garcia-Berrocoso T, Giralt D, Bustamante A, Montaner J. B-type natriuretic peptides (BNP and NT-proBNP) blood levels as biomarkers for all-cause mortality after stroke: a systematic review, meta-analysis and pooled-data analysis. PROSPERO 2012:CRD42012003284. Available at: http://www.crd.york.ac.uk/PROSPERO/display_record.
asp?ID=CRD42012003284\#.Umou6HDWMil. Accessed October 2013.

15. Moher D, Liberati A, Tetzlaff J, Altman DG; PRISMA Group. Preferred reporting items for systematic reviews and meta-analyses: the PRISMA statement. PLoS Med 2009;6:e1000097.

16. Duval S, Tweedie R. Trim and fill: a simple funnel-plotbased method of testing and adjusting for publication bias in meta-analysis. Biometrics 2000;56:455-463.

17. Efron B, Tibshirani R. An introduction to the bootstrap. In: Cox DR, Hinkley DV, Reid N, Rubin DB, Silverman BW, editors. Monographs on Statistics and Applied Probability. New York: Chapman \& Hall/CRC; 1993.

18. DeLong ER, DeLong DM, Clarke-Pearson DL. Comparing the areas under two or more correlated receiver operating characteristic curves: a nonparametric approach. Biometrics 1988;44:837-845.

19. Pencina MJ, D'Agostino RB, D'Agostino RB, Vasan RS. Evaluating the added predictive ability of a new marker: from area under the ROC curve to reclassification and beyond. Stat Med 2008;27:157-172.

20. Etgen T, Baum H, Sander K, Sander D. Cardiac troponins and $\mathrm{N}$-terminal pro-brain natriuretic peptide in acute ischemic stroke do not relate to clinical prognosis. Stroke 2005;36:270-275.

21. Sharma JC, Ananda K, Ross I, Hill R, Vassallo M. $\mathrm{N}$-terminal probrain natriuretic peptide levels predict short-term poststroke survival. J Stroke Cerebrovasc Dis 2006;15:121-127.

22. Koenig MA, Puttgen HA, Prabhakaran V, Reich D, Stevens RD. B-type natriuretic peptide as a marker for heart failure in patients with acute stroke. Intensive Care Med 2007;33:1587-1593.

23. Modrego PJ, Boned B, Berlanga JJ, Serrano M. Plasmatic $\mathrm{B}$-type natriuretic peptide and $\mathrm{C}$-reactive protein in hyperacute stroke as markers of CT-evidence of brain edema. Int J Med Sci 2008;5:18-23.

24. Idris I, Hill R, Ross I, Sharma JC. N-terminal probrain natriuretic peptide predicts 1-year mortality following acute stroke: possible evidence of occult cardiac dysfunction among patients with acute stroke. Age Ageing 2010; 39:752-755.

25. Cakir Z, Saritas A, Emet M, Aslan S, Akoz A, Gundogdu F. A prospective study of brain natriuretic peptide levels in three subgroups: stroke with hypertension, stroke without hypertension, and hypertension alone. Ann Indian Acad Neurol 2010;13:47-51.

26. Üstündağ M, Orak M, Güloğlu C, Öztürk E, Tamam Y, Kale E. The role of serum ferritin, pro-brain natriuretic peptide and homocysteine levels in determining ischaemic stroke subtype, severity and mortality. J Emerg Med 2010; 17:13-21.

27. Shibazaki K, Kimura K, Iguchi Y, Aoki J, Sakai K, Kobayashi K. Plasma brain natriuretic peptide predicts death during hospitalization in acute ischaemic stroke and transient ischaemic attack patients with atrial fibrillation. Eur J Neurol 2011;18:165-169.

28. Rost N, Biffi A, Cloonan L, et al. Brain natriuretic peptide predicts functional outcome in ischemic stroke. Stroke 2012;43:441-445.

29. Hajdinjak E, Klemen P, Grmec Š. Prognostic value of a single prehospital measurement of $\mathrm{N}$-terminal pro-brain natriuretic peptide and troponin $\mathrm{T}$ after acute ischaemic stroke. J Int Med Res 2012;40:768-776. 
30. Chen X, Zhan X, Chen M, et al. The prognostic value of combined NT-pro-BNP levels and NIHSS scores in patients with acute ischemic stroke. Intern Med 2012;51:2887-2892.

31. Stewart LA, Parmar MKB. Meta-analysis of the literature or of individual patient data: is there a difference? Lancet 1993;341:418-422.

32. Riley RD, Lambert PC, Abo-Zaid G. Meta-analysis of individual participant data: rationale, conduct, and reporting. BMJ 2010;340:c221.

33. McCullough PA, Omland T, Maisel AS. B-type natriuretic peptides: a diagnostic breakthrough for clinicians. Rev Cardiovasc Med 2003;4:72-80.

34. Kim SH, Lee JY, Park SH, et al. Plasma B-type natriuretic peptide level in patients with acute cerebral infarction according to infarction subtype and infarction volume. Int J Med Sci 2013;10:103-109.
35. Sharma JC, Ross I, Vassallo M. Cardio-protection in acute stroke. Int J Stroke 2007;2:288-290.

36. Montaner J, Perea-Gainza M, Delgado P, et al. Etiologic diagnosis of ischemic stroke subtypes with plasma biomarkers. Stroke 2008;39:2280-2287.

37. Santamarina E, Penalba A, García-Berrocoso T, et al. Biomarker level improves the diagnosis of embolic source in ischemic stroke of unknown origin. J Neurol 2012;259: 2538-2545.

38. Vuolteenaho O, Ala-Kopsala M, Ruskoaho H. BNP as a biomarker in heart disease. Adv Clin Chem 2005;40:1-36.

39. Srisawasdi P, Vanavanan S, Charoenpanichkit C, Kroll MH. The effect of renal dysfunction on BNP, NT-proBNP, and their ratio. Am J Clin Pathol 2010; 133:14-23.

\section{Target Your Job Search}

Your goal is precise, your time is precious. So give it your best shot. The AAN's Neurology Career Center is the largest neurology-specific job site tailored to in-demand neurology professionals like you.

Visit www.aan.com/careers and create your free profile today.

\section{You're Committed to Continually Expanding Your Knowledge}

NeuroSAETM is committed to helping you. The Sixth Edition of the AAN's convenient online selfassessment examination is now available, featuring 150 questions and 8 self-assessment CME credits upon successful completion to help meet ABPN MOC requirements.

See all the available NeuroSAE versions and purchase the exam today at AAN.com/view/neuroSAE.

Neurology ${ }^{\circledR}$ Online CME Program
subscribers. Simply read the articles marked CME, go to www.neurology.org, and click on CME.
This will provide all of the information necessary to get started. The American Academy of
Neurology (AAN) is accredited by the Accreditation Council for Continuing Medical Education
(ACCME) to sponsor continuing medical education for physicians. Neurology is planned and
produced in accordance with the ACCME Essentials. For more information, contact AAN Member
Services at $800-879-1960$.

